\title{
Existence of Solutions for Volterra Integro-Differential Equations with Implicit Derivative
}

\author{
Giuseppe Anichini ${ }^{1}$, Giuseppe Conti ${ }^{1}$ \\ ${ }^{1}$ Dipartimento di Matematica, e Informatica "U. Dini”, Università di Firenze, Firenze, Italy.
}

*Corresponding Author: Giuseppe Anichini, Dipartimento di Matematica, e Informatica "U. Dini", Università di Firenze, Firenze, Italy.

\begin{abstract}
The purpose of this paper is to study the existence of continuous solutions of an integro-differential equation with implicit derivative, on bounded intervals. In our investigations we aimed to extended the use of fixed-point theorems for uppersemicontinuous mapping with acyclic values in a Banach space to get the result.
\end{abstract}

Keywords: Metric spaces, multivalued maps, absolute retracts, cohomology, acyclic sets, integro-differential equations, functions equicontinuous and uniformly bounded, Ascoli-Arzelà theorem.

\section{INTRODUCTION}

In this paper we study, in an abstract setting, the solvability of a nonlinear integro-differential equation of Volterra type with implicit derivative like:

$$
x^{\prime}(t)=\int k(t, s) f\left(s, x(s), x^{\prime}(s)\right) d s, x(0)=0, t \in[0,1]
$$

We will look for solutions of these equations in the Banach space of all real $C^{1}$ functions which are defined in the real interval $[0,1]$.

Equation (1) is a special case of integro-differential equation. It is well known that the theory of integro-differential equations has been emerging as an important area of investigation in recent years and has been developed very rapidly due to the fact that such equations find a wide range of applications modelling adequately many real processes observed in physics, chemistry, biology, and engineering (see, e.g., [1], [2], [3], [4], [5], [6] and the recension therein).

Particularly, the integro-differential equations are involved through modelling in the framework of heat flow in material, kinetic theory, electrical engineering, vehicular traffic theory, biology, queuing theory, population dynamics, control theory, mathematical economics, mechanics. For example, Balachandran and Somasundaram (see [2]) proved an existence theorem for the optimal control of nonlinear system having an implicit derivative with quadratic performance involving an integrodifferential term also by using the same method of functional analysis, i. e. a fixed point theorem.

The integro-differential equations have been studied in various papers with the help of several tools of functional analysis, topology and fixed point theory. For instance, we can refer to [1], [2], [4], [5], [6], [7].

So, the crucial key of our approach in order to find solutions of equation (1) consists in the use of a very useful fixed point theorem for multivalued, compact, uppersemicontinuous maps, with acyclic values in a Banach space.

\section{Preliminaries ANd Notations}

Let $B=C^{1}\left(J, \mathbb{R}^{n}\right)$ be a Banach space of all continuously differentiable function defined on $J=[a, b]$ with the norm

$$
\begin{aligned}
& \|x\|_{1}=\max \left\{\|x\|,\left\|x^{\prime}\right\|\right\}, \quad \text { where } \quad\|x\|=\max \{|x(t)|, t \in[a, b]\} \\
& \left\|x^{\prime}\right\|=\max \left\{\left|x^{\prime}(t)\right|, t \in[a, b]\right\} .
\end{aligned}
$$


A subset $A \subset C^{1}\left(J, \mathbb{R}^{n}\right)$ is a relatively compact set if and only if the functions of $A$ are equicontinuous and uniformly bounded (together with their derivatives on $J$ ).

Let $M$ be a subset of the Banach space $B$ and let $T: M \rightarrow B$ be a map. Let $\left\{\varepsilon_{n}\right\}$ be an infinitesimal sequence of positive real numbers. A sequence $\left\{T_{n}\right\}$ of maps $T_{n}: M \rightarrow B$ is said to be an $\varepsilon_{n}$ approximation of $T$ if $\left\|T_{n}(x)-T(x)\right\|_{1} \leq \varepsilon_{n}$ for every $x \in M$.

Let us denote by $C(B)$ the family of all nonempty and compact subset of $B$ and denote by $B(0, r)$ the ball of $B$ defined by

$B(O, r)=\{x \in B:\|x\|<r\}$ (and by $\overline{B(O, r)}$ its closure).

Let $X$ be a subset of $B$; a multivalued map $S: X \rightarrow C(B)$ is said to be uppersemicontinuous (u. s. c.) if the graph of $S$ is closed, i.e. for any sequence $\left\{x_{n}\right\} \subset X, x_{n} \rightarrow x_{0}, y_{n} \rightarrow y_{0}$ as $n \rightarrow+\infty$, we have $y_{0} \in S\left(x_{0}\right)$.

A mapping from $B$ to $C(B)$ is said to be compact if it sends bounded sets into relatively compact sets.

We say that $A \subset B$ is an $R_{\delta}-$ set in the space $B$ if $A$ is the intersection of countable decreasing sequence of absolute retracts contained in $B$. It is known that $R_{\delta}$-set is an acyclic set, i.e. it is acyclic with respect to any continuous theory of cohomology (see for instance [8]).

Let $M$ be a closed and nonempty subset of $B$ and let $T: M \rightarrow B$ be a compact mapping. Let $T_{n}: M \rightarrow$ $B$ be an

$\varepsilon_{n}$ - approximation of $T$, where $T_{n}$ are compact mappings. It is known that, if the equation $x-T_{n}(x)$ $=y$ has at most one solution belonging to $\overline{B\left(0, \varepsilon_{n}\right)}$ for every natural number $n$, then the set of fixed points of $T$ is a compact $R_{\delta}-$ set (see [9]).

The well-known Gronwall Lemma, from the standard theory of Ordinary Differential Equations, will be used.

Proposition 1: Let $u, v, g: J: \rightarrow \mathbb{R}$ be continuous and nonnegative functions; moreover, assume that $g$ is a nondecreasing function on $J$. If the following inequality holds: $u(t) \leq g(t)+\int_{a}^{t} v(s) u(s) d s, t \in J$, then we have:

$u(t) \leq g(t) \exp \left(\int_{a}^{t} v(s) d s\right), t \in J$

The following proposition can be deduced from Theorem 1 of [10] and it will be useful in the sequel.

Proposition 2: Let $B$ be a Banach space, let $M$ be a closed and convex subset of $B$. Assume that $S: M \rightarrow C(B)$ be an uppersemicontinuous, compact multivalued map with acyclic values. Then, if $S(M) \subseteq M, S$ has a fixed point.

\section{MAIN RESUlt}

We want to deal with the existence of solutions of integro-differential equation (1).

The following theorem holds.

Theorem: Assume that the following conditions hold:

i) $\quad f:[0,1] \times \mathbb{R}^{n} \times \mathbb{R}^{n} \rightarrow \mathbb{R}^{n}$ is a continuous function such that, for every $(s, x, y) \in[0,1] \times$ $\mathbb{R}^{\mathrm{n}} \times \mathbb{R}^{\mathrm{n}}$, we have $|f(s, x, y)| \leq a|x|+b|y|, \quad a, b \geq 0$.

ii) $\quad k:[0,1] \times[0,1] \rightarrow \mathbb{R}^{n}$ is a $C^{l}$ function such that there exists a continuous nonnegative function

$h: J: \rightarrow \mathbb{R}$ satisfying the following conditions: 


$$
|k(t, s)| \leq h(s) \quad \text { and } \quad\left|\frac{\partial k}{\partial t}(t, s)\right| \leq h(s) \quad \text { for every }(t, s) \in[0,1] \times[0,1]
$$

iii) Putting $\bar{h}=\sup \{|h(s)|, s \in[0,1]\}$, the following inequality holds: $2 \bar{h} a+\bar{h} b<1$.

Then, if the conditions i), ii), iii) hold, equation (1) has at least one solution.

Proof. Let $q$ be a function belonging to $C^{1}\left(J, \mathbb{R}^{n}\right)$ and consider the following integral equation:

$$
y(t)=\int k(t, s) f\left(s, \int_{0}^{s} q(\tau) d \tau, y(s)\right) d s, t \in[0,1]
$$

Let $\Sigma: C^{1}\left(J, \mathbb{R}^{n}\right) \rightarrow C^{1}\left(J, \mathbb{R}^{n}\right)$ be the map which associates to every $q \in C^{1}\left(J, \mathbb{R}^{n}\right)$ the set of solutions of equation (3). Clearly, putting $x(t)=\int_{0}^{t} y(s) d s$, we have $x^{\prime}(t)=y(t)$ and $x(0)=0$; so that, the fixed points of the map $\Sigma$ are the solutions of equation (1).

To that aim, the following steps in the proof have to be established:

a) there exists a closed and convex set $C$ such that $\Sigma(C) \subseteq C$;

b) the map $\Sigma$ is an uppersemicontinuous and compact map;

c) the set $\Sigma(q)$ is an acyclic set for every $q \in C$.

a) Let $C=\overline{B(O, M}$ ) the closed ball of $C^{1}\left(J, \mathbb{R}^{n}\right)$; hence $y \in C$ if and only if $\|y\| \leq M$ and $\left\|y^{\prime}\right\| \leq M$

Let $q \in C$; we have:

$|y(t)| \leq\left|\int_{0}^{t} k(t, s) f\left(s, \int_{0}^{s} q(\tau) d \tau, y(s)\right) d s\right| \leq\left|\int_{0}^{t} h(s)\left(a\left|\int_{0}^{s} q(\tau) d \tau\right|+b|y(s)|\right) d s\right| \leq \bar{h} a\|q\|+\bar{h} b\|y\|$

So that we have: $\|y\| \leq \bar{h} a M+\bar{h} b\|y\|$.

Then it follows: $\|y\| \leq \frac{\bar{h} a M}{1-\bar{h} b}$; hence, $\|y\| \leq M$ if $\frac{\bar{h} a}{1-\bar{h} b}<1$.

Since $2 \bar{h} a+\bar{h} b<1$, then the last inequality holds.

Moreover, we have:

$$
y^{\prime}(t)=\int_{0}^{t} \frac{\partial k}{\partial t}(t, s) f\left(s, \int_{0}^{s} q(\tau) d \tau, y(s)\right) d s+k(t, t) f\left(t, \int_{0}^{t} q(s) d s, y(t)\right) .
$$

Hence, we obtain:

$$
\left\|y^{\prime}\right\| \leq \bar{h}(a M+b\|y\|)+\bar{h}(a M+b\|y\|)=2 \bar{h}(a M+b\|y\|) \leq 2 \bar{h}\left(a M+\frac{a b \bar{h} M}{1-\bar{h} b}\right)=2 \bar{h} \frac{a M}{1-\bar{h} b} .
$$

Thus, we have $\left\|y^{\prime}\right\| \leq M$ if $\frac{2 \bar{h} a}{1-\bar{h} b}<1$; since $2 \bar{h} a+\bar{h} b<1$, then the last inequality holds.

Then condition $\Sigma(C) \subseteq C$ is satisfied.

b) We want to prove that the map $\Sigma: C \rightarrow C$ is compact. Let $y \in C$ and fix $\varepsilon>0$. For any $t_{1}$, $t_{2} \in[0,1]$ we have: 


$$
\begin{aligned}
& \left|y^{\prime}\left(t_{2}\right)-y^{\prime}\left(t_{1}\right)\right|=\mid \int_{0}^{t_{2}} \frac{\partial k}{\partial t}\left(t_{2}, s\right) f\left(s, \int_{0}^{s} q(\tau) d \tau, y(s)\right) d s+k\left(t_{2}, t_{2}\right) f\left(t_{2}, \int_{0}^{t_{2}} q(s) d s, y\left(t_{2}\right)\right)- \\
& \int_{0}^{t_{1}} \frac{\partial k}{\partial t}\left(t_{1}, s\right) f\left(s, \int_{0}^{s} q(\tau) d \tau, y(s)\right) d s-k\left(t_{1}, t_{1}\right) f\left(t_{1}, \int_{0}^{t_{1}} q(s) d s, y\left(t_{1}\right)\right) \mid= \\
& \mid \int_{0}^{t_{1}} \frac{\partial k}{\partial t}\left(t_{2}, s\right) f\left(s, \int_{0}^{s} q(\tau) d \tau, y(s)\right) d s+ \\
& \left.+t_{2}, t_{2}\right) f\left(t_{2}, \int_{0}^{t_{2}} q(s) d s, y\left(t_{2}\right)\right)-k\left(t_{1}, t_{1}\right) f\left(t_{1}, \int_{0}^{t_{1}} q(s) d s, y\left(t_{1}\right)\right)-\int_{0}^{t_{1}} \frac{\partial k}{\partial t}\left(t_{1}, s\right) f\left(s, \int_{0}^{s} q(\tau) d \tau, y(s)\right) d s \\
& +\int_{t_{1}}^{t_{2}} \frac{\partial k}{\partial t}\left(t_{2}, s\right) f\left(s, \int_{0}^{s} q(\tau) d \tau, y(s)\right) d s \mid \leq \\
& \int_{0}^{t_{1}}\left|\frac{\partial k}{\partial t}\left(t_{2}, s\right)-\frac{\partial k}{\partial t}\left(t_{1}, s\right)\right|\left(a\left|\int_{0}^{s} q(\tau) d \tau\right|+b|y(s)| d s+\right. \\
& \bar{h} \mid f\left(t_{2}, \int_{0}^{t_{2}} q(s) d s, y\left(t_{2}\right)\right)-f\left(t_{1}, \int_{0}^{t_{1}} q(s) d s, y\left(t_{1}\right)|+\bar{h}| \int_{t_{1}}^{t_{2}} f\left(s, \int_{0}^{s} q(\tau) d \tau, y(s) \mid d s .\right.\right.
\end{aligned}
$$

By the continuity of the functions $q, h, f$ and $k$ it follows that there exists $\delta>0$ such that $\left|y^{\prime}\left(t_{2}\right)-y^{\prime}\left(t_{1}\right)\right|<\varepsilon$ for any $t_{1}, t_{2} \in[0,1],\left|t_{2}-t_{1}\right|<\delta$.

Since $|y(t)| \leq M$ and $\left|y^{\prime}(t)\right| \leq M$, it follows that the set $\Sigma(C)$ is (relatively) compact.

Let us now show that the multivalued map $\Sigma$ is uppersemicontinuous.

Let $\left\{q_{n}\right\}$ be a sequence converging to $q_{0}$ in the $C^{1}$-norm, $y_{n} \in \Sigma\left(q_{n}\right)$, i.e. let

$$
y_{n}(t)=\int_{0}^{t} k(t, s) f\left(s, \int_{0}^{s} q_{n}(\tau) d \tau, y_{n}(s)\right) d s, \quad t \in[0,1]
$$

Assume that $y_{n} \rightarrow y_{0}$ in the $C^{1}$-norm; we need to show that $y_{0} \in \Sigma\left(q_{0}\right)$.

From the Dominated Lebesgue Convergence Theorem it follows:

$$
\begin{aligned}
& \lim _{n \rightarrow+\infty} f\left(s, \int_{0}^{s} q_{n}(\tau) d \tau, y_{n}(s)\right) d s=f\left(s, \int_{0}^{s} q_{0}(\tau) d \tau, y_{0}(s)\right) d s \text { and } \\
& \lim _{n \rightarrow+\infty} y_{n}(t)=\lim _{n \rightarrow+\infty} \int_{0}^{t} k(t, s) f\left(s, \int_{0}^{s} q_{n}(\tau) d \tau, y_{n}(s)\right) d s=\int_{0}^{t} \lim _{n \rightarrow+\infty} k(t, s) f\left(s, \int_{0}^{s} q_{n}(\tau) d \tau, y_{n}(s)\right) d s= \\
& =\int_{0}^{t} k(t, s) f\left(s, \int_{0}^{s} q_{0}(\tau) d \tau, y_{\mathrm{O}}(s)\right) d s .
\end{aligned}
$$

Hence, we have:

$$
y_{0}(t)=\int_{0}^{t} k(t, s) f\left(s, \int_{0}^{s} q_{0}(\tau) d \tau, y_{0}(s)\right) d s \text {, i.e. } y_{0} \in \Sigma\left(q_{0}\right) .
$$

c) Now we want to show that, for every fixed $\bar{q} \in C$, the set $\Sigma(\bar{q})$ is an acyclic set.

Consider the following integral equation:

$$
y(t)=\int_{0}^{t} k(t, s) f\left(s, \int_{0}^{s} \bar{q}(\tau) d \tau, y(s)\right) d s, \quad t \in[0,1]
$$


Putting $f\left(s, \int_{0}^{s} \bar{q}(\tau) d \tau, y\right)=g(s, y)$, then the equation (4) can be written in the following way:

$$
y(t)=\int_{0}^{t} k(t, s) g(s, y(s)) d s, \quad t \in[0,1]
$$

Consider the operator $H: C \rightarrow C^{1}\left(J, \mathbb{R}^{n}\right)$ defined as follows:

$$
H(y)(t)=\int_{0}^{t} k(t, s) g(s, y(s)) d s, \quad t \in[0,1]
$$

Observe that the operator $H$ is compact. Clearly the fixed point of the operator (6) are the solutions of equation (5).

For every natural number $n$ there exists a Lipschitz function $g_{n}:[0,1] \times \mathbb{R}^{n} \rightarrow \mathbb{R}^{n}$ such that we have for every $(s, y)$,

$(s, z) \in[0,1] \times \mathbb{R}^{\mathrm{n}}:$

$$
\left|g_{n}(s, y)-g_{n}(s, z)\right| \leq L_{n}|y-z| \text { and }\left|g_{n}(s, y)-g(s, y)\right| \leq \frac{1}{2 n \bar{h}} \quad \text { (see [9]). }
$$

Let $H_{n}: C \rightarrow C^{1}\left(J, \mathbb{R}^{n}\right)$ be the operator defined as follows:

$$
H_{n}(y)(t)=\int_{0}^{t} k(t, s) g_{n}(s, y(s)) d s, \quad t \in[0,1]
$$

The operator $H_{n}$ defined in (7) is a compact operator for every natural number $n$. Moreover, we have:

$$
\begin{aligned}
& \left|H_{n}(y)(t)-H(y)(t)\right| \leq \int_{0}^{t}|k(t, s)|\left|g_{n}(s, y(s))-g(s, y(s))\right| d s \leq \frac{\dot{h}}{2 n h} \leq \frac{1}{n} \\
& \left|H^{\prime}{ }_{n}(y)(t)-H^{\prime}(y)(t)\right| \leq \\
& \left|\int_{0}^{t} \frac{\partial k}{\partial t}(t, s) g_{n}(s, y(s)) d s-\int_{0}^{t} \frac{\partial k}{\partial t}(t, s) g(s, y(s)) d s+k(t, t) g_{n}(t, y(t))-k(t, t) g(t, y(t))\right| \leq \\
& \int_{0}^{t} h(s)\left|g_{n}(s, y(s))-g(s, y(s))\right| d s+\bar{h}\left|g_{n}(t, y(t))-g(t, y(t))\right| \leq \frac{\overline{2 h}}{2 n \bar{h}}=\frac{1}{n} .
\end{aligned}
$$

Let now $\beta \in C$. Consider the equation $y-H_{n}(y)=\beta$; we want to prove that this equation has at most one solution.

Let $z$ be another solution; we have:

$$
|y(t)-z(t)| \leq \int_{0}^{t} h(s)\left|g_{n}(s, y(s))-g_{n}(s, z(s))\right| d s \leq L_{n} \int_{0}^{t} h(s)|y(s)-z(s)| d s .
$$

Using formula (2) we obtain: $|y(t)-z(t)| \leq 0$. Hence, $y(t)=z(t)$ for every $t \in[0,1]$.

Then we can conclude that the set $\Sigma(\bar{q})$ of the solutions of equation (4) is an acyclic set.

\section{EXAMPLe}

Let us consider the following integro-differential equation with implicit derivative:

$$
x^{\prime}(t)=\int_{0}^{t} \frac{\cos t}{s^{2}+4} \frac{\sin \left(x^{\prime}(s)\right)+\ln (|x(s)|+1)}{x^{2}(s)+1} d s, \quad x(0)=0, \quad t \in[0,1]
$$

By recalling our result, we have for every $(t, s) \in[0,1] \times[0,1]: \quad k(t, s)=\frac{\cos t}{s^{2}+4}$ and so $h(s)=\frac{1}{s^{2}+4} \leq \frac{1}{4}$.

In analogous way we have: $\left|\frac{\partial k}{\partial t}(t, s)\right| \leq h(s) \leq \frac{1}{4}$. So, we can put: $a=1, b=1, \bar{h}=\frac{1}{4}$.

Finally, the we obtain: $2 \bar{h} a+\bar{h} b=\frac{3}{4}<1$.

Then we can say that the conditions of our theorem are satisfied and that the equation (8) admits at least one solution. 


\section{REFERENCES}

[1] Anichini G., Conti G., Existence of solutions for Volterra integral equations depending on derivative, Pioneer Journal of Mathematics and Mathematical Sciences, 18(1), pp. 45-60 (2016).

[2] Balachandran K., Somasundaram D., Existence of optimal control for nonlinear systems with quadratic performance, J. Australian Math. Soc., Ser B, 29, pp. 249-255 (1987).

[3] Hoai N. T., K., Loi N. V., Existence of solutions for some Hammerstein type integro-differential inclusions, Electronic Journal of Differential Equations, 178, pp.1-8 (2007).

[4] Mallika Arjunan M., Selvi S., Existence results for impulsive mixed Volterra-Fredholm integro-differential inclusions with nonlocal conditions, Int. Journal of Mathematical Sciences and Applications, 1(1), Paper $n$. 6, $19(2011)$

[5] Pachpatte B. G., Applications of Leray-Schauder alternative to some Volterra integral and integrodifferential equations, Indian Journal Pure and Applied Mathematics, 26, pp. 1161-1168 (1995).

[6] Pachpatte B. G., Implicit type Volterra integro-differential equation, Tamkang Journal of Mathematics, 41(1), pp. 97-107 (2010).

[7] Sikorska A, Existence theory for nonlinear Volterra integral and differential equations, Journal Inequalities and Applications, 6, pp. 325-338 (2001).

[8] Gabor G., On the acyclicity of fixed point sets of multivalued maps, Topological Methods in Nonlinear Analysis, 14, pp. 327-343 (1999).

[9] Lasry J. M., Robert R.,Analyse non linéaire multivoque, Cahiers mathématiques de la decision, No. 7611, Paris (1976)

[10] Fitzpatrick P. M., Petryshin W. W., Fixed point theorems for multivalued noncompact acyclic mappings, Pacific Journal of Mathematics, 54(2), pp. 17-23 (1974).

Citation: G. Anichini, G. Conti, "Existence of Solutions for Volterra Integro-Differential Equations with Implicit Derivative ", International Journal of Scientific and Innovative Mathematical Research, vol. 6, no. 4, p. 10-15, 2018., http://dx.doi.org/10.20431/2347-3142.0604002

Copyright: (C) 2018 Authors. This is an open-access article distributed under the terms of the Creative Commons Attribution License, which permits unrestricted use, distribution, and reproduction in any medium, provided the original author and source are credited. 